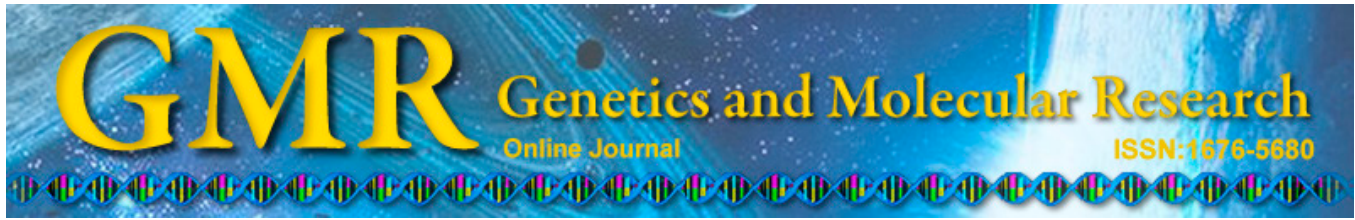

\title{
Molecular variation of Sporisorium scitamineum in Mainland China revealed by internal transcribed spacers
}

\author{
Y.Y. Zhang ${ }^{1,2 *}$, N. Huang ${ }^{1,2 *}$, X.H. Xiao ${ }^{1,2}$, L. Huang ${ }^{1,2}$, F. Liu ${ }^{1,2}$, W.H. Su ${ }^{1,2}$ \\ and Y.X. Que ${ }^{1,2}$ \\ ${ }^{1}$ Key Lab of Sugarcane Biology and Genetic Breeding, Ministry of Agriculture, \\ Fujian Agriculture and Forestry University, Fuzhou, Fujian, China \\ ${ }^{2}$ Sugarcane Research \& Development Center, \\ China Agriculture Research System \\ Fujian Agriculture and Forestry University, Fuzhou, Fujian, China \\ *These authors contributed equally to this study. \\ Corresponding author: Y.X. Que \\ E-mail: queyouxiong@126.com
}

Genet. Mol. Res. 14 (3): 7894-7909 (2015)

Received November 19, 2014

Accepted March 12, 2015

Published July 14, 2015

DOI http://dx.doi.org/10.4238/2015.July.14.15

\begin{abstract}
Sugarcane smut caused by the fungus Sporisorium scitamineum is a worldwide disease and also one of the most prevalent diseases in sugarcane production in mainland China. To study molecular variation in $S$. scitamineum, $23 \mathrm{~S}$. scitamineum isolates from the 6 primary sugarcane production areas in mainland, China (Guangxi, Yunnan, Guangdong, Hainan, Fujian, and Jiangxi Provinces), were assessed using internal transcribed spacer (ITS) methods. The results of ITS sequence analysis showed that the organisms can be defined at the genus level, including Ustilago and Sporisorium, and can also differentiate between closely related species. This method was not suitable for phylogenetic relationship analysis of different $S$. scitamineum isolates and could not provide support regarding their race ascription at the molecular level. The results of the present study will be useful for studies examining the molecular diversity of S. scita-
\end{abstract}


mineum and for establishing a genetic foundation for their pathogenicity differentiation and new race detection. In addition, our results can provide useful information for the pathogen selection principle in sugarcane smut resistance breeding and variety distribution.

Key words: Internal transcribed spacer; Molecular diversity; Sporisorium scitamineum; Sugarcane smut

\section{INTRODUCTION}

Sugarcane smut is a fungal disease caused by Sporisorium scitamineum. It is present worldwide and is one of the most prevalent diseases affecting sugarcane production in mainland China. This fungal disease has been reported in nearly every country in which sugarcane is planted, except for Papua New Guinea, where the ancestral domesticated species Saccharum officinarum originated. S. scitamineum causes serious loss in stalk yield and sucrose content in susceptible varieties (Hoy, 1986; Chao et al., 1990). As long as the smut appears in the sugarcane-growing area, it will produce enormous quantity of teliospores, making it difficult to completely eliminate this disease. In recent years, the genetic diversity of this fungus has been studied on a global scale (Braithwaite et al., 2004) and was found to be very limited outside of Asia. While the highest diversity is found in certain populations from Asia, that of populations in the Americas and Africa is extremely limited, and all non-Asian isolates were found to have originated from the same genotype (Braithwaite et al., 2004). Thus, cultivation of resistant varieties is a practical and economical method for controlling smut disease outside of Asia. In mainland China, given the variability of the pathogen, the genetic relationships between S. scitamineum varieties, particularly those in the 6 main sugarcane planting provinces (Guangxi, Yunnan, Guangdong, Hainan, Fujian, and Jiangxi), should be analyzed.

Although disease control can center on breeding smut-resistant varieties, the genetic basis for resistance remains unclear. Understanding the genetic diversity or fungal relationships would be helpful for determining the genetic basis of sugarcane smut resistance. Studies of fungi at the DNA level will increase the understanding of the genetic makeup as well as benefit smut resistance breeding and variety distribution. With the rapid development of molecular biology, several methods have been found to be useful for analyzing phylogenetic relationships and genetic variations in fungi (Henson, 1992; Pagel, 1999; Thomas and Richard, 2004). Molecular detection of the smut pathogen in sugarcane has become possible by using polymerase chain reaction (PCR) to amplify the bE mating-type gene of S. scitamineum (Byther and Steiner, 1974; Albert and Schenck, 1996; Schenck, 1998; Singh et al., 2004; Su et al., 2013). Random-amplified polymorphic DNA, amplified fragment length polymorphism, sequence-related amplified polymorphism, inter-simple sequence repeat, and internal transcribed spacer (ITS) sequence analysis have been used to evaluate intraspecies diversity within S. scitamineum isolates (Victoria et al., 1997; Bakkeren et al., 2000; Braithwaite et al., 2004; Stoll et al., 2003, 2005; Xu et al., 2004, 2014; Singh et al., 2005; Baraket et al., 2009; Que et al., 2012, 2014a,b). Therefore, molecular methods can be applied for phylogeny analysis because the phylogeny of S. scitamineum is poorly understood.

Ribosomal DNA (rDNA), which codes for ribosomal RNA, is organized into 3 genes coding for the ribosomal units and 2 ITS regions (Hibbett, 1992). Nuclear rDNA occurs as tandem repeat arrays with several hundred copies per genome, allowing for easy accessibility and amplification by PCR (White et al., 1990; Bruns et al., 1992). However, rDNA genes, 
such as many other multi-gene families, are subjected to concerted evolution, resulting in homogeneity among the different copies of the gene in the cell, making them ideal phylogenetic markers for phylogenetic analysis (Hillis and Dixon, 1991; Li, 1997; Page and Holmes, 1998). Therefore, the ITS region is a locus, which typically shows variation at the species level, and it is an important locus for phylogenetic analysis and particularly ecology. The multicopy arrangement and highly conserved priming sites in ITS make it easy to amplify from virtually all fungi, even when the material is marginal in quantity or quality. Moreover, the current and growing sequence data available for ITS enhance the value of this region (Martin, 2002; Thomas and Richard, 2004). ITS data can be informative at many taxonomic levels and frequently allows for distinction between organisms at the species level (Hibbett, 1992; Zambino and Szabo, 1993). Thus, nucleotide sequences of the rRNA gene have been widely examined (Henson, 1992; Pagel, 1999; Thomas and Richard, 2004). However, there have been no reports of the application of ITS sequence analysis to study the molecular variation of S. scitamineum in mainland China.

The objective of the present study was to comprehensively examine the molecular variation of the $S$. scitamineum pathogen isolated from the main sugarcane planting areas in mainland China using ITS. Our results will be helpful for understanding the variation and differentiation of this pathogen, as well as to further enrich genetic research studies of species. Moreover, our results provide information that can be used for pathogen selection principle in sugarcane smut resistance breeding and variety distribution.

\section{MATERIAL AND METHODS}

\section{Test materials}

Twenty-three sugarcane smut isolates (S. scitamineum) were collected from 6 main sugarcane-producing provinces in China. Single spores from smut whip were isolated and cultured for DNA extraction as described by Que et al. (2004). The codes and sources of these isolates are shown in Table 1.

\begin{tabular}{lll}
\multicolumn{2}{c}{ Table 1. Twenty-three isolates of Sporisorium Scitamineum used in this study. } & \\
\hline Code of isolate & Geographical origin & \\
\hline 1 & Fujian & Host (varieties) \\
2 & Fujian & Badila \\
3 & Fujian & Co1001 \\
4 & Fujian & F134 \\
5 & Fujian & NCo310 \\
6 & Fujian & NCo376 \\
7 & Fujian & Guitang946-119 \\
8 & Jiangxi & Guitang94-119 \\
9 & Jiangxi & Mintang95-354 \\
10 & Guangdong & Yuetang93-159 \\
11 & Guangdong & ROC16 \\
12 & Guangxi & ROC16 \\
13 & Guangxi & Guitang21 \\
14 & Hainan & Guitang16 \\
15 & Hainan & Yuetang2-126 \\
16 & Yunnan & CP34-85 \\
17 & Yunnan & Chuanzhe2 \\
18 & Yunnan & Yacheng55-1 \\
19 & Yunnan & Yacheng73-498 \\
20 & Yunnan & Yacheng84-37 \\
21 & Yunnan & Yuetang75-897 \\
22 & Yunnan & F172 \\
23 & Yunnan & ROC22 \\
\hline
\end{tabular}




\section{DNA extraction}

Mycelia derived from single spores were used in DNA extraction using the sodium dodecyl sulfate procedure described by Que et al. (2004). DNA was detected and quantified by $1.0 \%$ agarose gel electrophoresis.

\section{PCR identification of pathogen isolates}

All DNA samples isolated from the mycelia of putative S. scitamineum were subjected to PCR detection to verify S. scitamineum using primers bE4 and bE8, as described by Singh et al. (2004). Ten microliters from each PCR were subjected to electrophoresis, stained, and photographed under ultraviolet light. Distilled water was used as a negative control. To ensure that the primers only amplified the correct DNA sequences, amplified fragments were sequenced by Shanghai Generay Biotech Co., Ltd. (Shanghai, China). Sequences were then aligned with the published sequence (GenBank accession No. U61290).

\section{PCR amplification of ITS1 $\rightarrow$ ITS4 regions of rDNA}

PCR was conducted with DNA extracted from mycelia derived from single spores using the ITS1 $\rightarrow$ ITS4 primer pair. The primer sequences were as follows: ITS1, 5'-TCCGTAGGTGAACCTGCGG-3' and ITS4, 5'-TCCTCCGCTTA TTGATATGC-3'. Amplifications were conducted in a 25- $\mu \mathrm{L}$ PCR mixtures containing $2.5 \mu \mathrm{L}$ 10X PCR buffer (plus $15 \mathrm{mM}$ $\mathrm{MgCl}_{2}$ ), $0.5 \mu \mathrm{L} 10 \mathrm{mM}$ dNTPs, $0.2 \mu \mathrm{L}$ TaKaRa Ex Taq DNA polymerase (5 U/ $\mu \mathrm{L}$ ) (Shiga, Japan), $1.0 \mu \mathrm{L} 10 \mu \mathrm{M}$ ITS1 and ITS4 primers, $1.0 \mu \mathrm{L}$ DNA template (approximately $40 \mathrm{ng}$ ), and $\mathrm{ddH}_{2} \mathrm{O}$. PCR was carried out using an Eppendorf PCR (Eppendorf, Hamburg, Germany) under the following conditions: $94^{\circ} \mathrm{C}$ for $5 \mathrm{~min}$, and then 35 cycles of $94^{\circ} \mathrm{C}$ for $1 \mathrm{~min}, 55^{\circ} \mathrm{C}$ for $1 \mathrm{~min}$, and $72^{\circ} \mathrm{C}$ for $3 \mathrm{~min}$, and a final step at $72^{\circ} \mathrm{C}$ for $10 \mathrm{~min}$. Ten microliters of each PCR product were separated by $1.5 \%$ agarose gel electrophoresis. The targeted bands were then cut from the gel and subcloned into the PMD18-T vector, and the corresponding positive clones were sent for sequencing.

\section{Data analysis}

For each isolate, forward and reverse sequences were assembled and edited using the DNAMAN program. Sequences were aligned and edited, evaluated manually, and ambiguities were examined and edited. Eight sequences of the rDNA for $S$. scitamineum available from GenBank with accession Nos. AF135433, AY345007, DQ004829, DQ004830, DQ004831, DQ004832, DQ004833, and DQ004834 were used to ensure that the correct ITS sequences of the isolates had been obtained. In addition, the ITS sequence of Cintractia axicola (AY344967) was used as the outgroup. The Clustalx1.83 program was used to align all sequences from different sugarcane smut isolates ( 20 obtained in this study and 8 from a previous study), and 10 of relative fungus. The Mega molecular software (version 5.0) was used to remove all vacancy or missing data in the alignment results, analyze the base composition, GC content, and different sites of DNA sequence, and number of transitions (or transversions). The Mega software was also used to calculate genetic distances of ITS sequence with the Kimura2-parameter, to build the phylogenetic trees of ITS, ITS1, and ITS2 sequences by neighbor-joining method, and to test the confidence of tree branches using the bootstrap test. 


\section{RESULTS}

\section{PCR detection of pathogen isolates}

All DNA samples isolated were evaluated by agarose gel electrophoresis, followed by staining with ethidium bromide. The ratio of absorbance at $260 \mathrm{~nm}$ to that at $280 \mathrm{~nm}$ was $1.8-2.0$, indicating insignificant levels of contaminating proteins and polysaccharides and that the DNA was of high purity without degradation.

The results of all DNA samples examined by PCR amplification using primers bE4 and bE8 to determine the authenticity of $S$. scitamineum were described. The sequences of the PCR fragments amplified from 5 isolates, with the unique length of $420 \mathrm{bp}$, were highly identical (99-100\%) to that of the b East mating-type gene published in GenBank with accession No. U61290. The accession No. were EF185084, EF185085, EF185086, EF185087, and EU427310. However, changes in several bases were detected in these sequences. Further investigation is required to verify whether these changes account for the genetic variation between these isolates. Notably, using the same primers and PCR protocol, 1 fragment of 453 bp (GenBank accession No. EF185088) was also amplified from the isolate DNA. This may indicate the emergence of a new race in sugarcane.

\section{ITS-PCR amplification, ITS sequence base composition, and GC content}

The results for the electropherogram of the 23 isolates from ITS-PCR amplification of genomic DNA showed that each sample could amplify a clear band of approximately 750 bp. For all 23 isolates, 23 expected amplification products of $750 \mathrm{bp}$ were sent for sequencing; however, only 19 were successfully sequenced. There was also a different amplified product from the variety NCo376, which had a length of approximately $700 \mathrm{bp}$ (692 bp by sequencing). The NCBI GenBank accession No. of all 20 ITS sequences obtained in this study were as follows: EF185066, EF185067, EF185068, EF185069, EF185070, EF185071, EF185072, EF185073, EF185074, EF185075, EF185076, EF185077, EF185078, EF185079, EF185080, EF185081, EF185082, EF185083, EU427308, and EU427309 (Table 2). The marked ITS1 sequence, 5.8S sequence, and ITS2 sequence are shown in Figure 1.

The DNA sequences of the entire ITS regions (including the 5.8S rDNA) of sugarcane smut ranged from 654 to $669 \mathrm{bp}$. The 5.8S rDNA regions, of which the GC content for all isolates was $47.1 \%$, were too conservative to have the same length. However, the ITS 1 and ITS2 regions showed some differences in the products, with lengths ranging from 207 to 216 and 292 to $219 \mathrm{bp}$ and GC content ranging from 43.2 to 43.8 and 45.5 to $48.6 \%$, respectively (Table 2). Additionally, the GC content of the ITS2 region showed greater variability than the other regions. Importantly, the base composition and GC content of the 8 sequences of the ITS for S. scitamineum from the database are in line with these results (Table 2).

\section{ITS sequence mutation sites}

Figures 2 and 3 show the multiple-comparison results of ITS1 and ITS2 sequences by taking missing Gap process. As shown in Figures 2 and 3, several base substitutions and base transversions such as A-T, G-A, G-T, and G-C were found. All sites and mutation types for these ITS sequences occurred in the ITS1 and ITS2 sequences. Except for the ITS-NCo376(1) 
sequence, the other 19 ITS1 sequences contained 5 variable sites and 2 parsimony-informative sites (with lengths of $217 \mathrm{bp}$ ), and the same variable sites and parsimony-informative sites were identified in the ITS2 sequences (with lengths of $300 \mathrm{bp}$ through arranging). If all 20 sequences with variable sites were calculated, the results showed that 52 and 58 sites were variable in the ITS1 and ITS2 sequences, respectively. Overall, information obtained from the ITS1 sequences was similar to that from the ITS2 sequences.

Table 2. Sequence information of ITS $1 \rightarrow$ ITS4 amplification.

\begin{tabular}{|c|c|c|c|c|c|c|c|c|c|}
\hline No. & Sequence & Accession No. & $\begin{array}{c}\text { ITS } 1 \rightarrow \text { ITS } 4 \\
\text { (bp) }\end{array}$ & ITS1 (bp) & $\begin{array}{c}\text { ITS1 } \\
(\mathrm{G}+\mathrm{C}) \%\end{array}$ & $5.8 \mathrm{~S}(\mathrm{bp})$ & $\begin{array}{c}5.8 \mathrm{~S} \\
(\mathrm{G}+\mathrm{C}) \%\end{array}$ & ITS2 (bp) & $\begin{array}{c}\text { ITS2 } \\
(\mathrm{G}+\mathrm{C}) \%\end{array}$ \\
\hline 1 & ITS-NCo376(1).seq & EF185083 & 692 & 207 & 42.5 & 155 & 47.1 & 292 & 48.6 \\
\hline 2 & ITS-GD.seq & EF185079 & 714 & 215 & 43.7 & 155 & 47.1 & 297 & 46.1 \\
\hline 3 & ITS-Gui16.seq & EF185075 & 714 & 215 & 43.7 & 155 & 47.1 & 295 & 46.4 \\
\hline 4 & ITS-Badila.seq & EF185078 & 714 & 215 & 43.2 & 155 & 47.1 & 297 & 46.5 \\
\hline 5 & ITS-Co1001.seq & EF185077 & 714 & 215 & 43.7 & 155 & 47.1 & 297 & 46.1 \\
\hline 6 & ITS-JG94/119.seq & EF185071 & 715 & 216 & 43.5 & 155 & 47.1 & 297 & 46.1 \\
\hline 7 & ITS-NCo376(2).seq & EU427308 & 714 & 215 & 43.7 & 155 & 47.1 & 297 & 46.1 \\
\hline 8 & ITS-MT95/354.seq & EF185081 & 714 & 215 & 43.7 & 155 & 47.1 & 297 & 46.1 \\
\hline 9 & ITS-MT76/2.seq & EF185076 & 714 & 215 & 43.7 & 155 & 47.1 & 297 & 46.1 \\
\hline 10 & ITS-ROC16.seq & EF185082 & 714 & 215 & 43.7 & 155 & 47.1 & 297 & 46.1 \\
\hline 11 & ITS-GZ.seq & EF185080 & 714 & 215 & 43.7 & 155 & 47.1 & 297 & 46.1 \\
\hline 12 & ITS-F134.seg & EF185066 & 716 & 215 & 43.3 & 155 & 47.1 & 299 & 45.8 \\
\hline 13 & ITS-GX.seq & EF185070 & 716 & 215 & 43.8 & 155 & 47.1 & 299 & 45.5 \\
\hline 14 & ITS-Gui11.seq & EF185067 & 716 & 215 & 43.8 & 155 & 47.1 & 299 & 45.5 \\
\hline 15 & ITS-ROC22.seq & EU427309 & 716 & 215 & 43.3 & 155 & 47.1 & 299 & 45.5 \\
\hline 16 & ITS-NCo310.seq & EF185069 & 716 & 215 & 43.3 & 155 & 47.1 & 299 & 45.5 \\
\hline 17 & ITS-Ye92/126.seq & EF185072 & 715 & 215 & 43.3 & 155 & 47.1 & 298 & 45.6 \\
\hline 18 & ITS-BG94/119.seq & EF185073 & 715 & 215 & 43.3 & 155 & 47.1 & 298 & 45.6 \\
\hline 19 & ITS-CP34/85.seq & EF185074 & 715 & 215 & 43.3 & 155 & 47.1 & 298 & 45.6 \\
\hline 20 & ITS-CZ.seq & EF185068 & 716 & 215 & 42.8 & 155 & 47.1 & 299 & 45.5 \\
\hline 21 & ITS-DQ004829.seq & DQ004829 & 703 & 216 & 43.5 & 155 & 47.1 & 297 & 46.1 \\
\hline 22 & ITS-DQ004832.seq & DQ004832 & 724 & 216 & 43.5 & 155 & 47.1 & 296 & 46.6 \\
\hline 23 & ITS-DQ004831.seq & DQ004831 & 715 & 215 & 43.2 & 155 & 47.1 & 296 & 46.3 \\
\hline 24 & ITS-DQ004834.seq & DQ004834 & 714 & 214 & 43.4 & 155 & 47.1 & 297 & 45.8 \\
\hline 25 & ITS-DQ004830.seq & DQ004830 & 725 & 217 & 43.8 & 155 & 47.1 & 298 & 46.0 \\
\hline 26 & ITS-AF135433.seq & AF135433 & 677 & 215 & 43.7 & 155 & 47.1 & 297 & 46.1 \\
\hline 27 & ITS-AY345007.seq & AY345007 & 738 & 215 & 43.7 & 155 & 47.1 & 297 & 46.1 \\
\hline 28 & ITS-DQ004833.seq & DQ004833 & 725 & 217 & 43.8 & 155 & 47.1 & 299 & 45.8 \\
\hline
\end{tabular}

"1 20" sequences from the present study, "21 28" sequences from database.

ATGGATCATTTCGAGTGAAACCTTTTTCCGAGGTGTGGCTCGCACCTGTCTAACTAAA
CTGGGCTACCTATTTCAACACGGTTGCATCGGTTGGGTCTGCCAAACAGTGCACGAAA
GTACCTGTGGAGGCAGCCCGATAATCTACCAAAACACTTTTGATGATCTAGGATTTGA
AAGTATTTAACATTTTACGACTGGTAATGCGGTCGTCTAAAATCTAAAAAACAACTTTT
GGCAACGGATCTCTTGGTTCTCCCATCGATGAAGAACGCAGCGAATTGCGATAAGTAA
TGTGAATTGCAGAAGTGAATCATCGAATCTTTGAACGCACCTTGCGCTCCTTGCAGATC
TAATCTGGGGAGCATGCCTATTTGAGGGCCGCGAATTGTTTCGAACGCATGCTTTTTT
ATTACGAAAGAGCTGGCGGATCGGTAGTGAGGGTTTTGCCATTTACCGTGGCTCCCTCG
AAATGCATTAGTGCATCCATTTGACAGGCAAAGACGGACGAAGGCTCGACTTTTGCC
CATCTTCCCTGCCAGGTTTTGATAATATCAGGGCTTTGGTGGTGAGGATGAGCAAGAA
GCTGGACGCGACGGCCTTTGCTGATTGGAGTGCTTCTGAACACCGCCCTAGCTAATTTT
TTTATTTTATTTTTTTGGCTAGGGAATAATTCAAATCGGCCTCAGATAGGTAGGACTAC
CCGCTGAACTTAA(NCBI Accession Number: EF185066)

Figure 1. Sequence of ITS1 $\rightarrow$ ITS4 amplification from sugarcane variety F134 collected in Fuzhou. Dotted underlined letters $=$ sequence of ITS1; shaded letters $=$ sequence of $5.8 \mathrm{~S}$; underlined letters $=$ sequence of ITS2. 


\begin{tabular}{|c|c|}
\hline & AGTGAAACCTTTTTTCCGAGGTGTGGCTCGCACCTGTCTAACTAAACT-GGGCTACCTATTTCAACACGGTTGCAT \\
\hline IS-Guil1.seq & 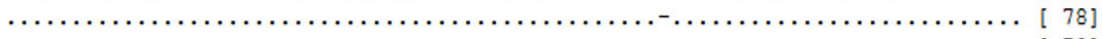 \\
\hline$-\mathrm{NCO} 310$ & 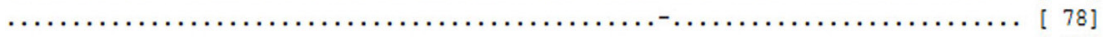 \\
\hline -Roc22.seq & $\ldots \ldots \ldots \ldots \ldots \ldots \ldots \ldots \ldots \ldots \ldots \ldots \ldots$ \\
\hline -Ye92/126.seq & 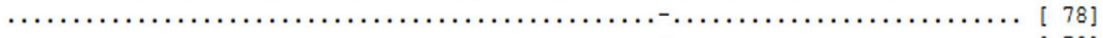 \\
\hline -BG94/119.seq & 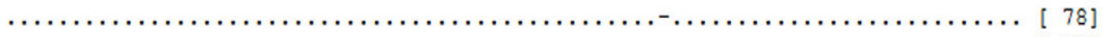 \\
\hline $\mathrm{CP} 34 / 85$. seq & 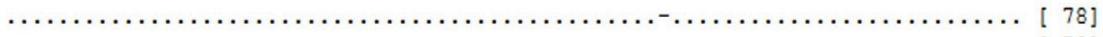 \\
\hline -Guil6.seq & 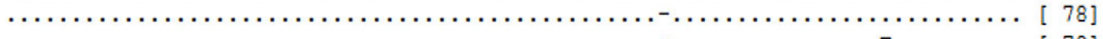 \\
\hline -Badila.seq & 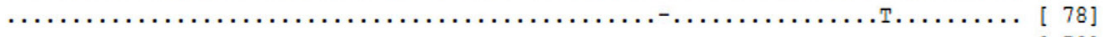 \\
\hline$-M T 76 / 2 . \operatorname{seq}$ & 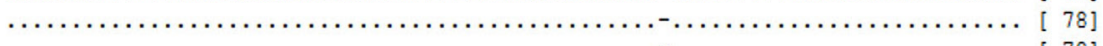 \\
\hline S-Co1001.seq & 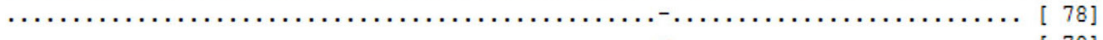 \\
\hline S-MT95/354.seq & 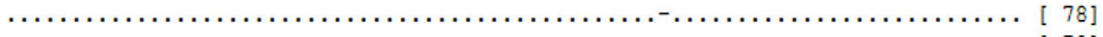 \\
\hline S-RoC16.seq & 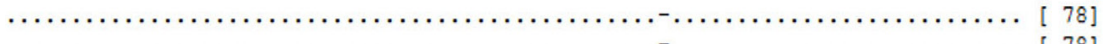 \\
\hline TS-GZ.seq & 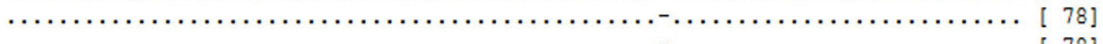 \\
\hline TS-GD.seq & 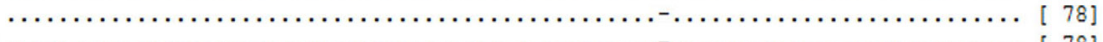 \\
\hline S-NC0376(2) .seq & 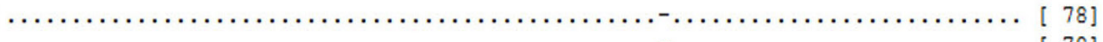 \\
\hline TS-JG94/119.seq & 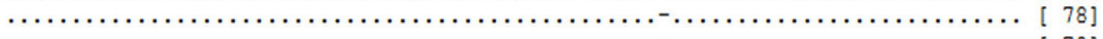 \\
\hline TS-GX.seq & 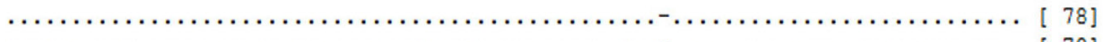 \\
\hline TS-CZ.seq & 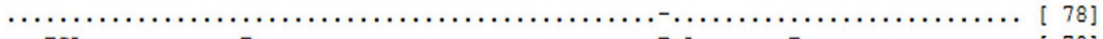 \\
\hline TS-NCO376(1) .seq & 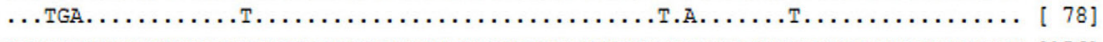 \\
\hline ITS-F134.seq & CGGTTGGGTCTGCCAAACAGTGCACGAAAGTACCTGTGGAGGCAGCCCGATAATCTACCAAAACACTTTTGATGATCT \\
\hline TS-Guil1.seq & $\cdots \cdots \cdots \cdots \cdots \cdots \cdots \cdots \cdots \cdots \cdots \cdots \cdots \cdots \cdots \cdots \cdots$ \\
\hline TS-NCO310.seq & 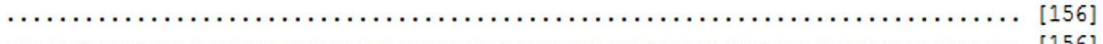 \\
\hline ITS-ROC22.seq & (2. \\
\hline TS-Ye92/126.seq & n \\
\hline TS-BG94/119.seq & $\cdots \cdots \cdots \cdots \cdots \cdots \cdots \cdots \cdots \cdots \cdots \cdots \cdots \cdots$ \\
\hline ITS-CP34/85.seq & $\cdots \cdots \cdots \cdots \cdots \cdots \cdots \cdots \cdots \cdots \cdots \cdots \cdots \cdots$ \\
\hline ITS-Guil6.seq & 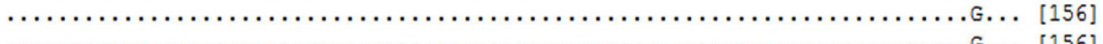 \\
\hline IS-Badila.seq & 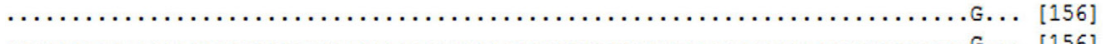 \\
\hline ITS-MT76/2.seq & 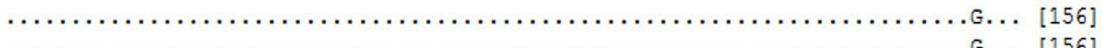 \\
\hline ITS-Co1001.seq & 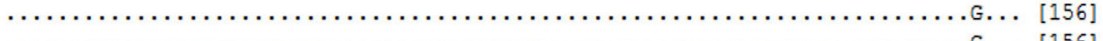 \\
\hline ITS-MT95/354.seq & 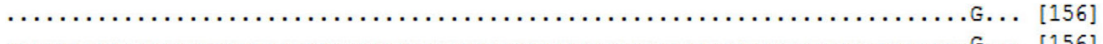 \\
\hline ITS-ROC16.seq & 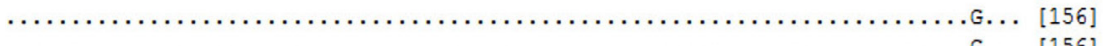 \\
\hline ITS-GZ.seq & 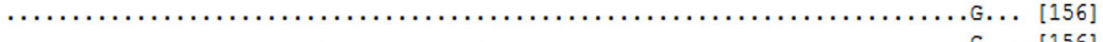 \\
\hline ITS-GD.seq & 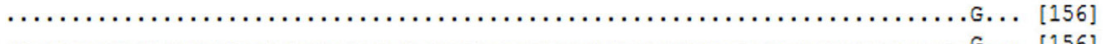 \\
\hline ITS-NC0376(2) . seq & 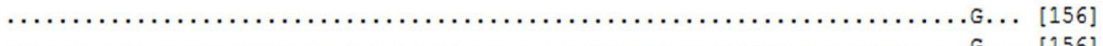 \\
\hline ITS-JG94/119.seq & \\
\hline ITS-GX.seq & 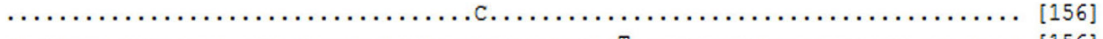 \\
\hline ITS-CZ.seq & \\
\hline ITS-NCO376(1) . seq & ...A.A.A.G..G..CGC.C.TT.TTGGACGTGA.CTC-.C.CT.T.T.C.С.T..AC. \\
\hline ITS-F134.seq & AGGATTTGAAA-GTATTTAACATTTTACGACTGGTAATGCGGTCGTCTAAAATCTAAAAAA [217] \\
\hline ITS-Guil1.seq & 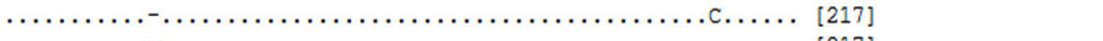 \\
\hline ITS-NCO310.seq & 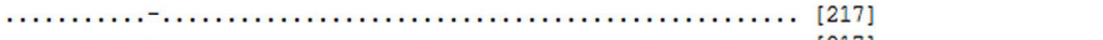 \\
\hline ITS-ROC22.seq & 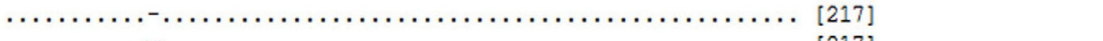 \\
\hline ITS-Ye92/126.seq & 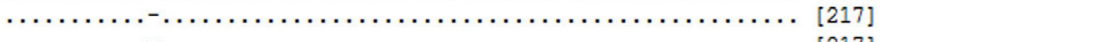 \\
\hline ITS-BG94/119.seq & 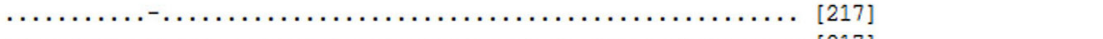 \\
\hline ITS-CP34/85.seq & 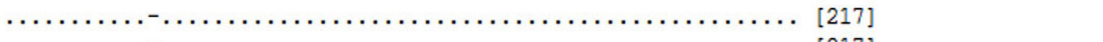 \\
\hline ITS-Guil6.seq & 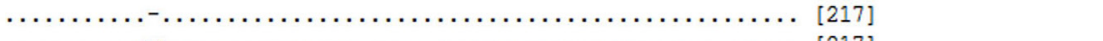 \\
\hline ITS-Badila.seq & 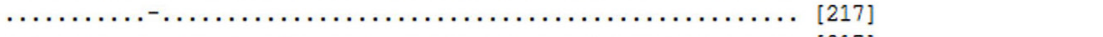 \\
\hline $\operatorname{ITS}-\mathrm{MT} 76 / 2 . \operatorname{seq}$ & 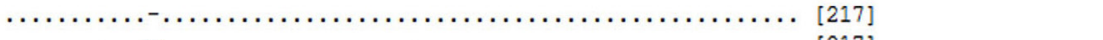 \\
\hline ITS-Co1001.seq & 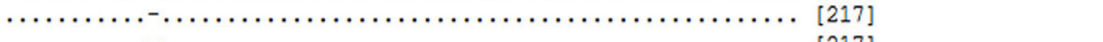 \\
\hline ITS-MT95/354.seq & 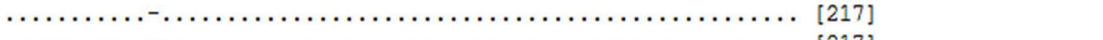 \\
\hline ITS-ROC16.seq & 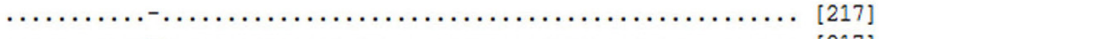 \\
\hline ITS-GZ.seq & 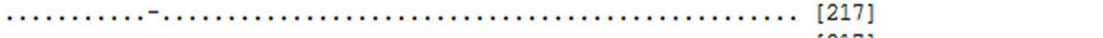 \\
\hline ITS-GD.seq & 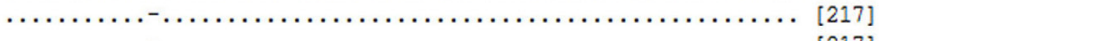 \\
\hline ITS-NC0376(2) .seq & 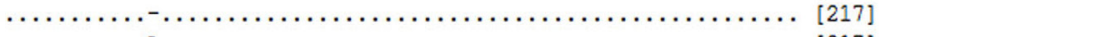 \\
\hline ITS-JG94/119.seq & 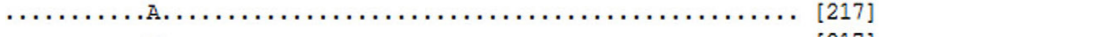 \\
\hline S-GX.seq & 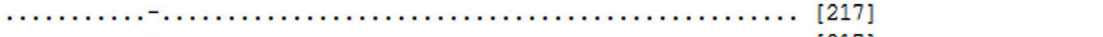 \\
\hline ITS-CZ.seq & 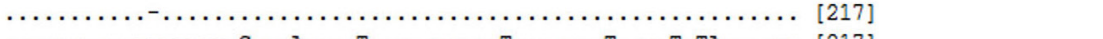 \\
\hline $\mathrm{S}-\mathrm{NCO} 376(1) \cdot 3$ & 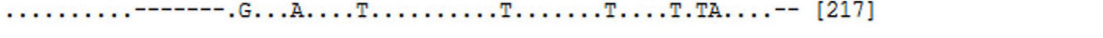 \\
\hline
\end{tabular}

Figure 2. ITS1 sequences of 20 sugarcane smut samples. Computer alignment of the ITS1 consensus sequences used MEGA5.0. Dots = base similarity; dashes $=$ deletion . 


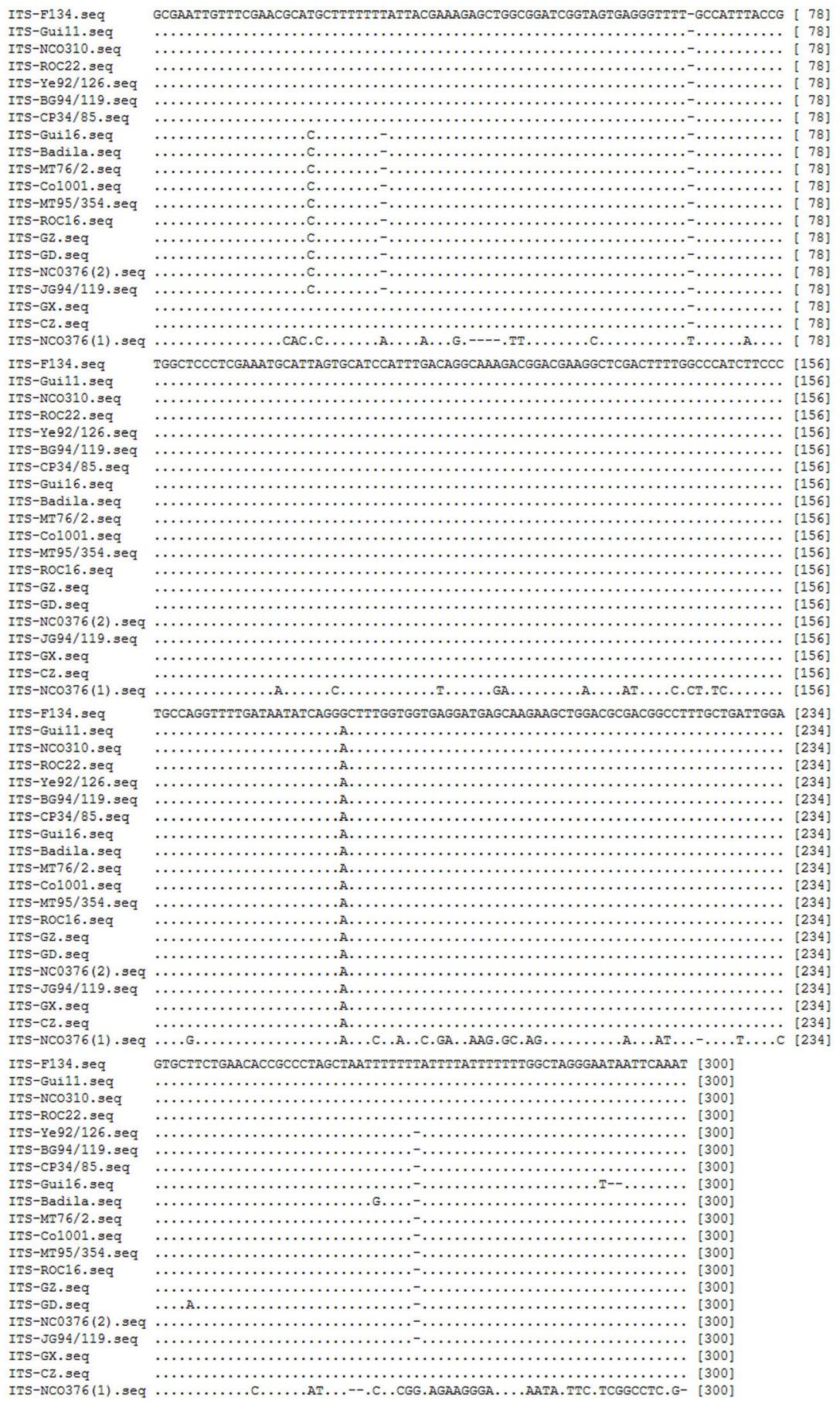

Figure 3. ITS2 sequences of 20 sugarcane smut samples. Computer alignment of the ITS 2 consensus sequences used MEGA5.0. Dots = base similarity; dashes = deletion. 


\section{Genetic distance and phylogenetic analysis of ITS1 $\rightarrow$ ITS4 aligned sequences}

By multiple comparison, using the MEGA5.0 software with the Kimura2-parameter, the genetic distance matrix between the different ITS sequences of $S$. scitamineum from different sugarcane varieties was generated. As shown in Table 3, the areas of genetic distance were 0.000 , which are highlighted in yellow. This result reflects that these pairs of sequences were very similar or that the ITS sequences were completely consistent. The raw data did not explain whether genetic distance of the sugarcane smut ITS sequences were associated with sugarcane varieties or origins. In addition, the calculated genetic distances between No. 1 (ITS-NCo376(1).seq) and other ITS sequences $(0.178-0.184)$ were significantly greater than the distances obtained by comparing other sequences with each other $(0.000-0.007)$.

Table 3. Genetic distance analysis of the ITS1 $\rightarrow$ ITS4 aligned sequences.

\begin{tabular}{|c|c|c|c|c|c|c|c|c|c|c|c|c|c|c|c|c|c|c|c|c|}
\hline No. & 1 & 2 & 3 & 4 & 5 & 6 & 7 & 8 & 9 & 10 & 11 & 12 & 13 & 14 & 15 & 16 & 17 & 18 & 19 & 20 \\
\hline 1 & & 0.182 & & & & & & & & & & & & 0.178 & 0.178 & 0.178 & 0.178 & 0.178 & 0.178 & 0.180 \\
\hline 2 & 0.182 & & & & & & & & & & & & & & & & & & & \\
\hline 3 & .182 & 0.003 & & 004 & & & & & & & & & & & & & & & & \\
\hline 4 & 184 & .004 & .004 & & & & & & & & & & & & & & & & & \\
\hline 5 & & 3 & 003 & 0.004 & & & 1 & & & & & & & & & & & & & 00 \\
\hline 6 & & & 001 & 0.003 & 0.001 & & 0.000 & & & & & & & & & & & & & .00 \\
\hline 7 & & & & 0 & .001 & 0 & & 0.000 & & & & & & & & & & & & \\
\hline 8 & & & & & & 000 & & & 0.00 & & & & & & & & & & & \\
\hline 9 & 0 & 1 & 001 & 003 & & 000 & 0.000 & 0.000 & & .000 & 00 & & & & & & & & & 0.004 \\
\hline 10 & 0.180 & 001 & & & & & 0.00 & & & & 0.000 & & & & & & & & & 0.004 \\
\hline 11 & 0.180 & 001 & 001 & .003 & & 0.000 & 0.000 & $0.0 \mathrm{C}$ & 0.000 & 0.000 & & 0.004 & & & & & & & & 0.00 \\
\hline 12 & 0.180 & 006 & 006 & .007 & 0.006 & 0.004 & 0.004 & 0.00 & & & 0.004 & & 0.004 & & & & & & & 0.003 \\
\hline 13 & 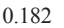 & .007 & 007 & 0 & & 0.006 & 0.006 & 0.006 & & & 0.006 & 0.004 & & 0.003 & & & & & & 0.00 \\
\hline 14 & & .004 & & 0 & 0.004 & 0.003 & 0.003 & 0.003 & 0.003 & & 0.003 & 0.001 & 0.003 & & 0.000 & 0.000 & 0.0 & & & 0.001 \\
\hline 15 & & .004 & & 0 & & 0.003 & 0.0 & & & & & 0.001 & & & & 0.000 & 0.0 & & & 0.001 \\
\hline & & & & & & & & & & & & & & & & & 0.0 & & & 0.00 \\
\hline & & & & & & & & & & & & & & & & & & & & \\
\hline & & & & & & & & & & & & & & & & & & & 0.00 & 0.0 \\
\hline 19 & & & & & & & & & & & & & & & & & & & & 0 \\
\hline 20 & 180 & 0.006 & 0.006 & 0.007 & 0.006 & 0.004 & 0.004 & 0.004 & 0.004 & 0.004 & 0.004 & 0.003 & 0.004 & 0.001 & 0.001 & 0.001 & 0.001 & 0.001 & 0.001 & \\
\hline
\end{tabular}

"1 20" sequences name refer to Table 2 .

Data were aligned in 3 ways, including the entire consensus ITS sequence of each isolate, the ITS1 region, and the ITS2 region. Using the MEGA5.0 program, 3 phylogenetic trees were generated, which showed that the cluster results derived from ITS sequence differed from those of the ITS1 and ITS2 regions (Figures 4 to 6).

For phylogenetic analysis of the ITS regions, except for the isolate ITS-NCo376(1). seq for which the ITS sequence length was $692 \mathrm{bp}$, the other 27 ITS sequences with lengths of approximately 750 bp formed 2 groups (99\% bootstrapping support). However, for phylogenetic analysis of both the ITS1 and ITS2 regions, these 27 ITS sequences (except for ITSNCo376(1).seq) formed 3 groups (99\% bootstrapping support) and 2 groups (100\% bootstrapping support). A higher bootstrapping support rate indicated a more stable clade. For the ITS, ITS1, and ITS2 regions, phylogenetic analysis showed that 38 sequences were divided into 2 clusters, but there was a large difference sequence in specific clades. In addition, the 27 ITS sequences were grouped closest to each other with a highest sequence similarity. Particularly, the ITS-NCo376(1).seq showed many differences from the other 27 ITS sequences from sugarcane smut fungus in all 3 clustering analyses. For the phylogenetic analysis of both the ITS 
and ITS2 regions, ITS-NCo376(1).seq had a higher identity with 4 groups of Sporisorium spp than that with 6 groups of Ustilago spp; the ITS-NCo376(1).seq formed a group with 6 groups of Ustilago spp in ITS1 sequence clustering analysis.

However, in all 3 cases, firstly, 28 ITS sequences of sugarcane smut fungus, got together for a group, the sugarcane smut fungus had a close kinship with Sporisorium fungi and a distant relationship with Ustilago fungi. The 4 types of Sporisorium spp formed one group, and the other Ustilago spp plus the ITS-NCo376(1).seq, of which the ITS sequence length was $692 \mathrm{bp}$, formed a separate group. These 2 groups showed low sequence similarity with each other (30-60\% bootstrapping support rate). Similar sequence identity was observed among all S. scitamineum isolates and 4 Sporisorium spp (surpasses $90 \%$ bootstrapping support), except for the isolate termed ITS-NCo376(1).seq, indicating that these isolates represented a single lineage and that the $U$. scitaminea should be replaced by $S$. scitamineum.

These results suggest that ITS analysis could only distinguish from different species of fungi and cannot be used to determine sequence difference among microspecies of smut strains with close genetic relationships. However, further studies are needed to confirm this.

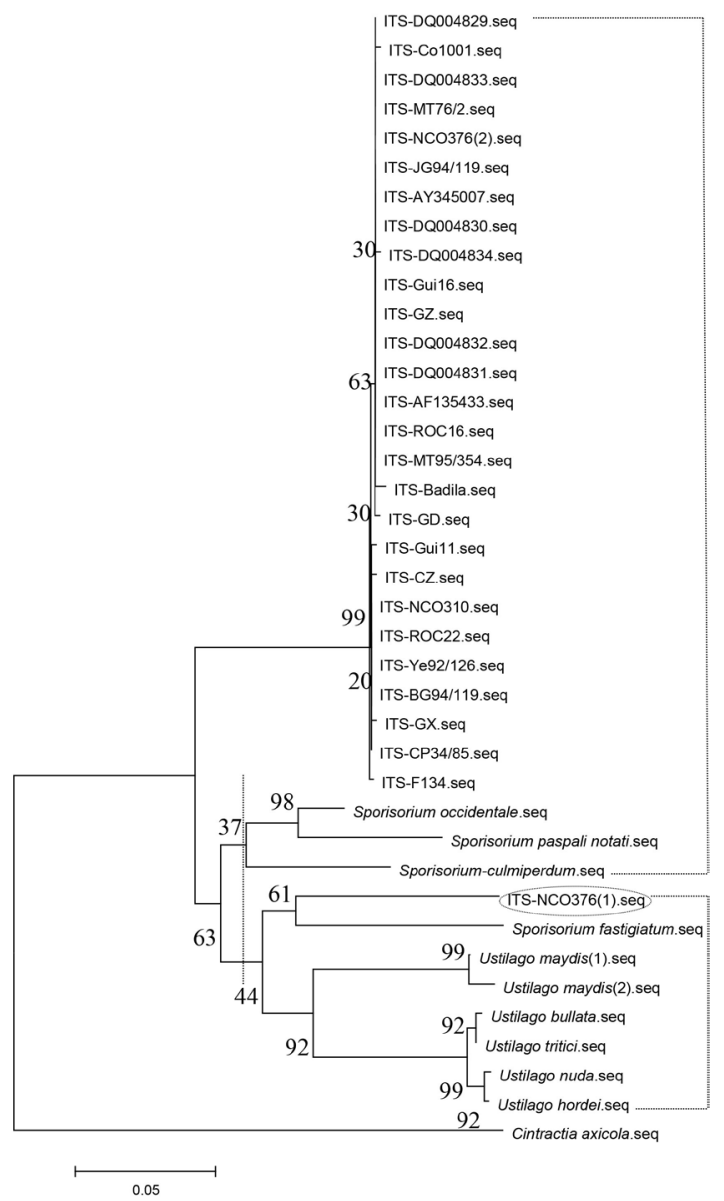

Figure 4. Clustering of the whole sequences of ITS. 


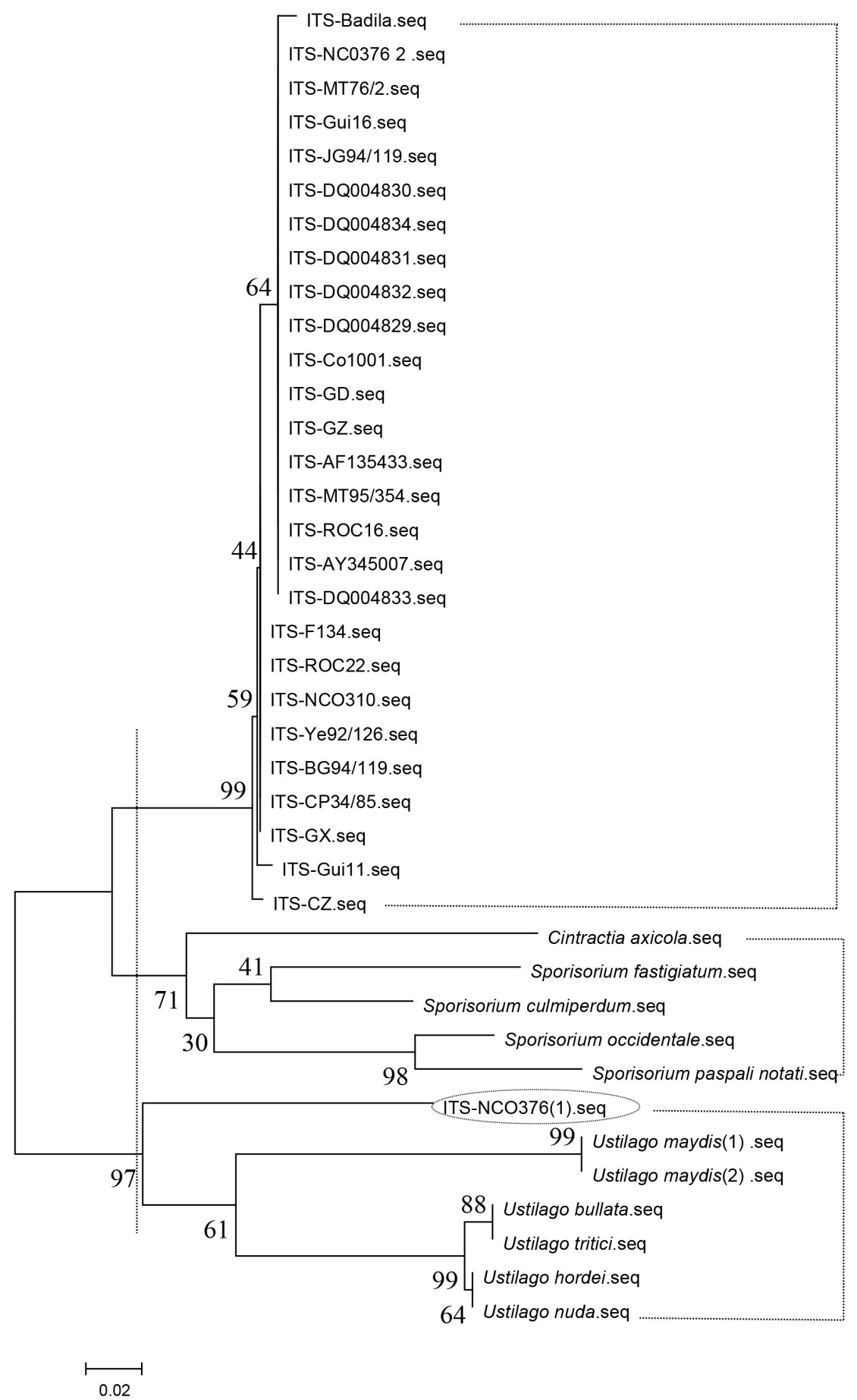

Figure 5. Clustering of the sequences of ITS1. 


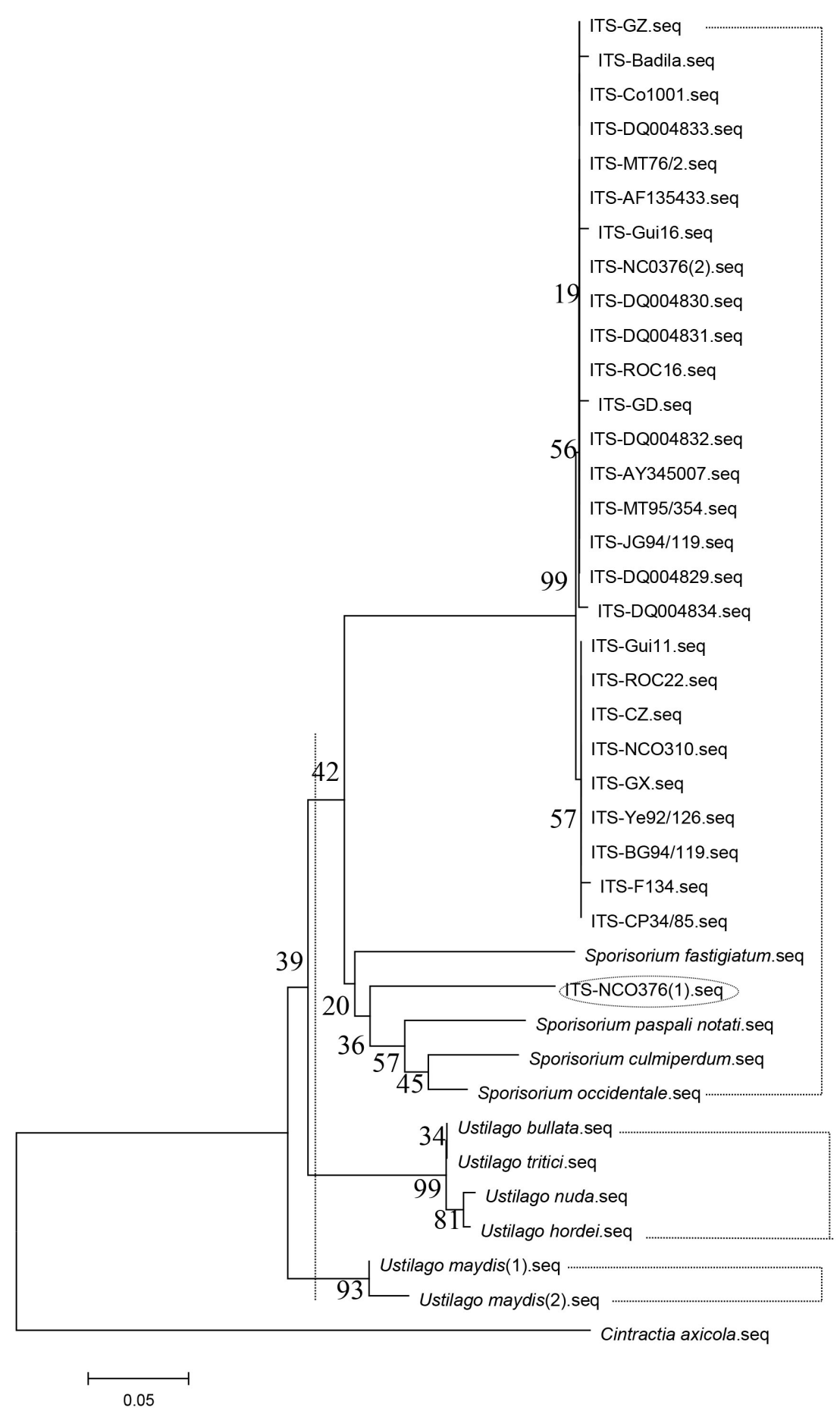

Figure 6. Clustering of the sequences of ITS2. 


\section{DISCUSSION}

rDNA is a source of detailed genetic information because changes in nucleotide sequences and the variation between different species are abundant. Compared with mitochondrial DNA, rDNA, which is protected by nuclear protection mechanism, undergoes a stable evolution process, and the spacer regions of ITS1 and ITS2 (18S, 5.8S, 26S rDNA) are very conserved and can be amplified by universal primers (Bruns et al., 1992; Gardes and Bruns, 1993). Therefore, ITS sequences appear to provide higher resolution at the species or subspecies level (Bruns et al., 1992; Gardes and Bruns, 1993). In the present study, ITS sequences of rDNA incorporating the ITS1, 5.8S, and ITS2 regions contained some variable sites ( 52 sites in ITS1 sequences and 58 sites in ITS2 sequences) and different variation types. Genetic information in ITS1 sequences was similar to that in ITS2 sequences, and thus both play important roles in taxonomic research.

ITS sequence data are typically used to define organisms at the genus level and resolve relationships between closely related species (Gardes et al., 1991; Bakkeren et al., 2000; Somai et al., 2002; Stoll et al., 2003). This was observed following comparison of sequence data from $S$. scitamineum isolates used in this study with those of published sequences for other members of Sporisorium and Ustilago (Table 3 and Figures 4 to 6). As indicated in the phylogenetic analysis, all ITS sequences tested were distinctly divided into 2 clades, Sporisorium and Ustilago. ITS, ITS1, and ITS2 sequence data revealed little variation among the sugarcane smut group (Figures 4 to 6 ). The nearly 100\% sequence identity of all S. scitamineum isolates, except the isolate from NCo376 named ITS-NCo376(1), was also confirmed in the genetic distance analysis. This demonstrates that this population represents a single lineage.

Interestingly, the economically important smut on sugarcane, S. scitamineum, appears to be part of the Sporisorium clade. Dendrograms constructed from ITS, ITS1, and ITS2 sequences indicate that an affiliation of the sugarcane smut with a well-supported Sporisorium clade, which agrees with the results of a previous study (Gardes et al., 1991; Bakkeren et al., 2000), but differs from the traditional classification which placed the sugarcane smut into the Ustilago clade. S. scitamineum shows morphological traits typical of Sporisorium species (Vanky, 1991, 2000; Bakkeren et al., 2000). The affiliation of S. scitamineum with Sporisorium was described in a recently published analysis of LSU and morphological data (Bakkeren et al., 2000; Piepenbring et al., 2002), which is also revealed in the taxonomic placement of $S$. scitamineum and genome synteny with $S$. reilianum, $U$. maydis, and $U$. hordei (Que et al., 2014a). However, whether this resulted from inappropriate nomination or only the conventional name, remains putative until reliable morphological traits agree with the results of extensive molecular studies. Importantly, with a unique length of $692 \mathrm{bp}$, the ITS termed ITS-NCo376(1) (GenBank accession No. EF185083) showed some different characteristics from the other 27 ITS sequences from S. scitamineum isolates. It is unclear whether this is a new race emerging or the result of increased variation. Further morphological and molecular researches are necessary to confirm this.

It is generally recognized that the most of the smut isolate from sugarcane variety F134 belongs to Race 2 and that NCo310 belongs to Race 1. Additionally, Race 3 has been reported in Chinese Taiwan in sugarcane variety ROC22, indicating that the isolate collected from ROC22 belongs to Race 1, Race 2, or Race 3. However, with $100 \%$ sequence identity, the 3 ITS sequences amplified from these isolates could not provide support regarding their race ascription at the molecular level. In addition, through ITS analysis, we could not determine the 
relationship between $S$. scitamineum and its geographical origin or host origin. Moreover, because of conservation in the ITS region, 2 ITS sequences (EF185083 and EF185083) derived from NCo376 and Badila, both of which were highly resistant to sugarcane smut, were similar to the other ITS sequences from different host origins. Therefore, intraspecific heterogeneity is a serious limitation for phylogenetic studies using the ITS region, but that worked fine-tune their choice of ITS sequence to some certain problems, such as phylogenetic relationships at the genus level. Additionally, in closely related isolates obtained from the same host, such as smut isolates from sugarcane, a significant number of altered base pairs in the ITS region may not clearly distinguish them, as was observed in this study. Other genes or DNA regions known to be more hypervariable, such as ribosomal polymerase B and the nuclear small subunit rRNA, must be analyzed.

Effective smut disease management strategies and successful acquirement of smut resistance require a clear understanding of variation in the pathogen population. For the mainland China sugar industry, it is critical to understand whether the sugar industry harbors different races of $S$. scitamineum so that the sugarcane breeding programs for smut resistance can target resistance to those races. To date, several races of $S$. scitamineum are known to exist, but different races are poorly understood and defined. Particularly, the actual number of smut races and their prevalence are unknown (Ferreira and Comstock, 1989). Classification of $S$. scitamineum is based mainly on differences in spore morphology and the characteristics of germinating spores. Additionally, sugarcane smut races can be classified according to the inoculation test of standard control varieties, and races are only indicated or suggested when a sugarcane cultivar succumbs to smut for several years (James, 1969; Lee-Lovick, 1978; Que et al., 2012). Although pathogenic variation was observed worldwide, only Taiwan showed evidence of distinct races. In this study, in addition to 21 isolates, 2 S. scitamineum isolates (Codes 4 and 3 in Table 1), which were assumed to be Races 1 and 2, were collected from the sugarcane varieties $\mathrm{NCO} 310$ and $\mathrm{F} 134$, respectively. The aim was to acquire information regarding the molecular differentiation among the 23 S. scitamineum isolates collected from 6 main sugarcane-producing areas through ITS sequence analysis, as well as molecular support for race classification. Nevertheless, the 23 isolates occupied here could not be divided into 2 clusters based on Race 1 and Race 2 in ITS sequence analysis. Race classification using the ITS analysis method was still unpractical. Moreover, although the S. scitamineum isolate (Code 23 in Table 1) was from ROC22, which was the first host of Race 3 reported in Chinese Taiwan, there were only limited base pair changes in the 23 isolates examined by ITS sequence analysis. Whether this represents a new race requires further investigation.

In conclusion, the molecular variation of $23 \mathrm{~S}$. scitamineum isolates was comprehensively investigated at the DNA level for 3 different regions using ITS methods. Our results will not only enrich studies examining the molecular diversity of $S$. scitamineum, but also provide a genetic foundation for their pathogenicity differentiation and new race detection. In contrast, if one isolate was found to be distinctly different from all the others, host identification should be applied to detect and validate its pathogenicity and determine a new race or isolate with further variation. Finally, these results suggest that gene flow between continents was rare, as a single lineage that originated in Asia had spread worldwide, likely through the accidental transfer of infected cuttings. Outside of Asia, the use of resistant varieties should thus be a sustainable and effective method of controlling the disease. In Asia, because of pathogen variability, the available resistance sources should be more clearly characterized, particularly those in wild species $S$. officinarum. 


\section{ACKNOWLEDGMENTS}

Research supported by the Natural Science Foundation of Fujian Province, China (\#2015J06006), the National Natural Science Foundation of China (\#31340060), the Program for New Century Excellent Talents in Fujian Province University (\#JA14095), the Research Funds for Distinguished Young Scientists in Fujian Provincial Department of Education (\#JA13090), and the Research Funds for Distinguished Young Scientists in Fujian Agriculture and Forestry University (\#xjq201202). We appreciate the ideas and constructive criticism provided by the reviewers.

\section{REFERENCES}

Albert HH and Schenck S (1996). PCR amplification from a homolog of the bE mating-type gene as a sensitive assay for the presence of Ustilago scitaminea DNA. Plant Dis. 80: 1189-1192.

Bakkeren G, Kronstad JW and Levesque CA (2000). Comparison of AFLP fingerprints and ITS sequences as phylogenetic markers in Ustilaginomycetes. Mycologia 92: 510-521.

Baraket G, Saddoud O, Chatti K, Trifi M, et al. (2009). Sequence analysis of the internal transcribed spacers (ITSs) region of the nuclear ribosomal DNA (nrDNA) in fig cultivars (Ficus carica L.). Sci. Horticult. 120: 34-40.

Braithwaite KS, Bakkeren G, Croft BJ and Brumbley SM (2004). Genetic variation in a worldwide collection of the sugarcane smut fungus Ustilago scitaminea. Proc. Aust. Soc. Sugar Cane Technol. 26: 1-9.

Bruns TD, Vilgalys R, Barns SM, Gonzalez D, et al. (1992). Evolutionary relationships within the fungi: analyses of nuclear small subunit rRNA sequences. Mol. Phylogenet. Evol. 1: 231-241.

Byther RS and Steiner GW (1974). Comparison of inoculated of techniques for smut disease testing in Hawaii. In: International Society Sugar Cane Technologists, 15th edn. (Dick J and Collingwood DJ, eds.). ISSCT, Durban, 280-288.

Chao CP, Hoy JW and Martin FA (1990). Evaluation of traits associated with resistance to sugarcane smut caused by Ustilago scitaminea. J. Am. Soc. Sugar Cane Technol. 9: 6-16.

Ferreira SA and Comstock JC (1989). Smut. In: Diseases of Sugarcane-Major Disease (Ricaud C, Egan BT, Gillaspie AG Jr and Hughes CG, eds.). Elsevier, Amsterdam, 211-229.

Gardes M and Bruns TD (1993). ITS primers with enhanced specificity for basidiomycetes - application to the identification of mycorrhizae and rusts. Mol. Ecol. 2: 113-118.

Gardes M, White TJ, Fortin J, Bruns TD, et al. (1991). Identification of indigenous and introduced symbiotic fungi in Ectomycorrhizae by amplification of nuclear and mitochondrial ribosomal DNA. Aust. J. Bot. 69: 180-190.

Henson JM (1992). DNA hybridization and polymerase chain reaction (PCR) tests for identification of Gaeumannomyces, Phialophora and Magnaporthe isolates. Mycol. Res. 96: 629-636.

Hibbett DS (1992). Ribosomal RNA and fungal systematics. Trans. Mycol. Soc. Jpn. 33: 533-556.

Hillis DM and Dixon MT (1991). Ribosomal DNA-molecular evolution and phylogenetic inference. Q. Rev. Biol. 66: 410-453.

Hoy JW (1986). Incidence of sugarcane smut in Louisiana and its effects on yield. Plant Dis. 70: 59-60.

James GL (1969). Smut susceptibility testing of sugarcane in Rhodesia. Proc. South Africa Sugar Technol. Assoc. 43: 85-92. Li WH (1997). Molecular Evolution. Sinauer, Sunderland.

Lee-Lovick G (1978). Smut of sugarcane Ustilago scitaminea. Plant Pathol. 47: 181-188.

Martin AP (2002). Phylogenetic approaches for describing and comparing the diversity of microbial communities. Appl. Environ. Microbiol. 68: 3673-3682.

Page DM and Holmes EC (1998). Molecular Evolution: A Phylogenetic Approach. Blackwell Publishing Ltd., Hoboken.

Pagel M (1999). Inferring the historical patterns of biological evolution. Nature 401: 877-884.

Piepenbring M, Stoll M and Oberwinkler F (2002). The generic position of Ustilago maydis, Ustilago scitaminea, and Ustilago esculenta (Ustilaginales). Mycol. Prog. 1: 71-80.

Que YX, Zou TT and Xu LP (2004). The screening of medium for sugarcane Ustilago scitaminea and the technique for the isolation of its genomic DNA. Acta Agric. Univ. Jiangxiensis 26: 353-355.

Que YX, Xu LP, Lin JW, Chen RK, et al. (2012). Molecular variation of Sporisorium scitamineum in Mainland China revealed by RAPD and SRAP markers. Plant Dis. 96: 1519-1525.

Que YX, Xu LP, Wu QB, Liu YF, et al. (2014a). Genome sequencing of Sporisorium scitamineum provides insights into 
the pathogenic mechanisms of sugarcane smut. BMC Genomics 15: 996.

Que YX, Su YC, Guo JL, Wu QB, et al. (2014b). A global view of transcriptome dynamics during Sporisorium scitamineum challenge in sugarcane by RNA-seq. PLoS One 9: e106476.

Schenck S (1998). Evaluation of a PCR amplification method for detection of systemic smut infections in sugarcane. Sugar Cane 6: 2-5.

Singh N, Somai BM and Pillay D (2004). Smut disease assessment by PCR and microscopy in inoculated tissue cultured sugarcane cultivars. Plant Sci. 167: 987-994.

Singh N, Somai BM and Pillay D (2005). In vitro screening of sugarcane to evaluate smut susceptibility. Plant Cell Tissue Organ Cult. 80: 259-266.

Somai BM, Dean RA, Farnham MW, Zitter TA, et al. (2002). Internal transcribed spacer regions 1 and 2 and random amplified polymorphic DNA analysis of Didymella bryoniae from related Phoma species isolated from cucurbits. Phytopathology 92: 997-1004.

Stoll M, Piepenbring M, Begerow D and Oberwinkler F (2003). Molecular phylogeny of Ustilago and Sporisorium species (Basidiomycota, Ustilaginales) based on internal transcribed spacer (ITS) sequences. Can. J. Bot. 81: 976-984.

Stoll M, Begerow D and Oberwinkler F (2005). Molecular phylogeny of Ustilago, Sporisorium, and related taxa based on combined analyses of rDNA sequences. Mycol. Res. 109: 342-356.

Su YC, Wang SS, Guo JL, Xue BT, et al. (2013). A TaqMan Real-time PCR assay for detection and quantification of Sporisorium scitamineum in sugarcane. Sci. World J. 2013: Article ID 942682.

Thomas DB and Richard PS (2004). Evolutionary studies of ectomycorrhizal fungi: recent advances and future directions. Can. J. Bot. 82: 1122-1132.

Vanky K (1991). Spore morphology in the taxonomy of Ustilaginales. T. Mycol. Soc. Jpn. 32: 381-400.

Vanky K (2000). Taxonomical studies on Ustilaginales. XX. Mycotaxon 74: 161-215.

Victoria JI, Sánchez A and Guzmán ML (1997). Molecular characterization of cultures of sugarcane (Ustilago scitaminea) sporidia from two geographic areas of Columbia. In: Vth ISSCT Pathology Workshop (Abstract), KwaZulu-Natal.

White TJ, Bruns TD, Lee S and Taylor JW (1990). Amplification and direct sequencing of fungal ribosomal RNA genes for phylogenetics. In: PCR protocols: a guide to methods and applications (Innis MA, Gelfand DH, Sninsky JJ, and White T, eds.) Academic Press, Waltham. 315-322.

$\mathrm{Xu}$ LP, Chen RK and Chen PH (2004). Analysis on infection index of smut caused by Ustilago scitaminea in sugarcane segregated population. Chin. J. Trop. Crops 25: 33-36.

Xu LP, Lu YH, You Q, Liu XL, et al. (2014). Biogeographical variation and population genetic structure of Sporisorium scitamineum in Mainland China: insights from ISSR and SP-SRAP markers. Sci. World J. 2014: Article ID 296020.

Zambino PL and Szabo LJ (1993). Phylogenetic relationships of selected cereal and grass based on rDNA sequence analysis. Mycologia 85: 401-414. 